\title{
PROPERLY DISTRIBUTED SUBSEQUENCE ON THE LINE
}

\section{A.I. ABDULNAGIMOV, A.S. KRIVOSHEYEV}

\begin{abstract}
In the article we consider first order sequences of complex numbers. We prove that a sequence of nonzero minimal density contains a subsequence of the same density. We also prove that a real sequence of nonzero minimal density contains a properly distributed subsequence. Basing on this fact, we prove a result on representation of an entire function of exponential type with real zeros as a product of two entire functions with the same properties. Moreover, one of these functions has a regular growth. As a corollary, we obtain a result on completeness of exponential systems with real exponents in the space of analytic functions in a bounded convex domain of the complex plane.
\end{abstract}

Keywords: entire function, regular growth, zero set

Mathematics Subject Classification: 30D10

\section{INTRODUCTION}

In the paper we mostly study real sequences of first order. We find the conditions under which such sequences contain a properly distributed set of a prescribed density. On the basis of these conditions we prove the result on representing an entire function of exponential type with real zeroes by a product of two functions of the same type, one of those has the a regular growth. As a corollary, we obtain the result on the completeness of exponential systems with real exponents in the space of functions analytic in a bounded convex planar domain.

Let $\Lambda=\left\{\lambda_{k}\right\}_{k=1}^{\infty}$ be a sequence of complex numbers taken in the ascending order of its absolute values. At that we assume that it can be multiple, i.e. some of $\lambda_{k}$ can coincide. We denote by $n(r, \Lambda)$ the number of the terms in sequence $\Lambda$ located in the circle $|\lambda|\langle r, r\rangle 0$. The lower and upper densities of $\Lambda$ are respectively the quantities

$$
\underline{n}(\Lambda)=\lim _{r \rightarrow \infty} \frac{n(r, \Lambda)}{r}, \quad \bar{n}(\Lambda)=\varlimsup_{r \rightarrow \infty} \frac{n(r, \Lambda)}{r} .
$$

Sequence $\Lambda$ is said to have density $n(\Lambda)$ if $\underline{n}(\Lambda)=\bar{n}(\Lambda)=n(\Lambda)$. It is easy to see that in this case the identity

$$
n(\Lambda)=\lim _{k \rightarrow \infty} \frac{k}{\left|\lambda_{k}\right|}
$$

holds true.

Maximal and minimal densities of sequence $\Lambda$ are the quantities

$$
\bar{n}_{0}(\Lambda)=\varlimsup_{\delta \rightarrow 0} \varlimsup_{r \rightarrow \infty} \frac{n(r, \Lambda)-n((1-\delta) r, \Lambda)}{\delta r}, \quad \underline{n}_{0}(\Lambda)=\lim _{\delta \rightarrow 0} \lim _{r \rightarrow \infty} \frac{n(r, \Lambda)-n((1-\delta) r, \Lambda)}{\delta r} .
$$

Lemma 1. Let $\Lambda=\left\{\lambda_{k}\right\}_{k=1}^{\infty}$ be such $\bar{n}(\Lambda)<\infty$. The inequalities

$$
\underline{n}_{0}(\Lambda) \leqslant \underline{n}(\Lambda) \leqslant \bar{n}(\Lambda) \leqslant \bar{n}_{0}(\Lambda)
$$

hold true.

A.I. Abdulnagimov, A.S. Krivosheyev, Properly distributed subsequence on the line.

(c) Abdulnagimov A.I., Krivosheyev A.S. 2015.

Submitted July 8, 2014. 
Proof. We have

$$
\begin{aligned}
\bar{n}_{0}(\Lambda) & =\varlimsup_{\delta \rightarrow 0} \varlimsup_{r \rightarrow \infty} \frac{n(r, \Lambda)-n((1-\delta) r, \Lambda)}{\delta r} \geqslant \varlimsup_{\delta \rightarrow 0}\left(\varlimsup_{r \rightarrow \infty} \frac{n(r, \Lambda)}{\delta r}-\varlimsup_{r \rightarrow \infty} \frac{n((1-\delta) r, \Lambda)}{\delta r}\right) \\
& =\varlimsup_{\delta \rightarrow 0}\left(\frac{\bar{n}(\Lambda)}{\delta}-(1-\delta) \varlimsup_{r \rightarrow \infty} \frac{n((1-\delta) r, \Lambda)}{(1-\delta) \delta r}\right)=\varlimsup_{\delta \rightarrow 0}\left(\frac{\bar{n}(\Lambda)}{\delta}-(1-\delta) \frac{\bar{n}(\Lambda)}{\delta}\right)=\bar{n}(\Lambda) .
\end{aligned}
$$

Thus, $\bar{n}(\Lambda) \leqslant \bar{n}_{0}(\Lambda)$.

Inequalities $\underline{n}(\Lambda) \leqslant \bar{n}(\Lambda)$ follow directly from the definitions of these quantuties.

To prove $(1)$, it remains to show that $\underline{n}_{0}(\Lambda) \leqslant \underline{n}(\Lambda)$. Let $\delta \in(0,1)$. We have

$$
\begin{aligned}
& \lim _{r \rightarrow \infty} \frac{n(r, \Lambda)-n((1-\delta) r, \Lambda)}{\delta r} \leqslant \lim _{r \rightarrow \infty} \frac{n(r, \Lambda)}{\delta r}-(1-\delta) \lim _{r \rightarrow \infty} \frac{n((1-\delta) r, \Lambda)}{\delta r} \\
& =\frac{\underline{n}(\Lambda)}{\delta}-(1-\delta) \frac{\underline{n}(\Lambda)}{\delta}=\underline{n}(\Lambda) \text {. }
\end{aligned}
$$

It implies the required inequality. The proof is complete.

Let $\Lambda=\left\{\lambda_{k}\right\}_{k=1}^{\infty}$ and $\widetilde{\Lambda}=\left\{\tilde{\lambda}_{n}\right\}_{n=1}^{\infty}$. If $\Lambda$ is a subsequence of $\widetilde{\Lambda}$, we shall write $\Lambda \subset \widetilde{\Lambda}$. The proof of the following statement is based on the approach employed in the proof of Lemma 5 in work [1].

Lemma 2. Let $\tau \geqslant 0$ and $\widetilde{\Lambda}=\left\{\tilde{\lambda}_{n}\right\}_{n=1}^{\infty}$ be such that $\underline{n}_{0}(\widetilde{\Lambda}) \geqslant \tau$. Then there exists a sequence $\Lambda \subset \widetilde{\Lambda}$ having density $\tau$.

Proof. Since the arguments of the terms in $\widetilde{\Lambda}$ makes no influence of its various densities, we can assume that sequence $\widetilde{\Lambda}$ lies on a non-negative real semi-axis. We can also assume that $\tau>0$, since the case $\tau=0$ is trivial.

Let $\alpha=1 / \tau$, and $\widetilde{\Lambda}_{m}$ be the set of all the terms in $\widetilde{\Lambda}$ belonging the semi-interval $[(m-1) \alpha, m \alpha), m \geqslant 1$. We seek sequence $\Lambda$ as the union $\Lambda=\bigcup_{m \geqslant 1} \Lambda_{m}$, where $\Lambda_{m}$ is a subset of $\widetilde{\Lambda}_{m}$. We construct sets $\Lambda_{m}, m \geqslant 1$, by induction following the restriction: for each $m \geqslant 1$ the total amount of points in sets $\Lambda_{1}, \ldots, \Lambda_{m}$ should be less or equal $m$.

Let $m=1$. If $\widetilde{\Lambda}_{1}$ is non-empty, we choose an arbitrary point $\tilde{\lambda}_{n(1)}$ in $\widetilde{\Lambda}_{1}$ and let $\lambda_{1}=\tilde{\lambda}_{n(1)}$, $\Lambda_{1}=\left\{\lambda_{1}\right\}$. Otherwise we let $\Lambda_{1}=\emptyset$. By construction, the number of points in set $\Lambda_{1}$ does not exceed one.

Assume that we have constructed sets $\Lambda_{m}$ for each $m<p$ and the number of points in these sets satisfy the aforementioned restriction. Let us define $\Lambda_{p}$. If total amount of points in sets $\Lambda_{1}, \ldots, \Lambda_{p-1}, \widetilde{\Lambda}_{p}$ is less or equal $p$, as $\Lambda_{p}$ we take the set $\widetilde{\Lambda}_{p}$. Otherwise, as $\Lambda_{p}$ we choose an arbitrary subset $\widetilde{\Lambda}_{p}$ such that the total amount of potins in sets $\Lambda_{1}, \ldots, \Lambda_{p}$ is $p$. Hence, the aforementioned restriction is satisfied.

Let us show that our construction is well-defined, i.e., at the last step in set $\widetilde{\Lambda}_{p}$ there are always a necessary amount of points. Suppose this is not true. In other words, for each subset $\Lambda_{p}$ (including an empty set) of set $\widetilde{\Lambda}_{p}$, the total amount of points in $\Lambda_{1}, \ldots, \Lambda_{p}$ is not $p$. Then for each $\Lambda_{p} \subset \widetilde{\Lambda}_{p}$ the total amount of points in $\Lambda_{1}, \ldots, \Lambda_{p}$ is either strictly less than $p$ or strictly greater than $p$. The first case is impossible since on the last step of construction it is assumed for $\Lambda_{p}=\widetilde{\Lambda}_{p}$ that the total amounts of points in $\Lambda_{1}, \ldots, \Lambda_{p}$ is strictly greater than $p$. The second case is also impossible since by the assumption of the induction the total amount of points in sets $\Lambda_{1}, \ldots, \Lambda_{p-1}$ is less or equal $p-1$, and therefore, for $\Lambda_{p}=\emptyset$ the total amount of points in $\Lambda_{1}, \ldots, \Lambda_{p}$ is also less than $p$. Thus, our construction is well-defined.

Let us show that $\Lambda$ is the sought set, i.e., $\Lambda \subset \widetilde{\Lambda}$ and $n(r, \Lambda) / r \rightarrow \tau$ as $r \rightarrow \infty$. The former is valid by the construction. Let us show the latter. Let $r>0$ and $q(r)$ stands for the maximal natural number satisfying the inequality $\alpha q(r) \leqslant r$. By construction, the quantity 
$n(\alpha(q(r)+1), \Lambda)$ coincides with the total amount of point in sets $\Lambda_{1}, \ldots, \Lambda_{q(r)+1}$; in accordance with the above restriction this amount does not exceed $q(r)+1$. Therefore,

$$
\bar{n}(\Lambda)=\varlimsup_{r \rightarrow \infty} \frac{n(r, \Lambda)}{r} \leqslant \varlimsup_{r \rightarrow \infty} \frac{n(\alpha(q(r)+1), \Lambda)}{\alpha q(r)} \leqslant \varlimsup_{r \rightarrow \infty} \frac{q(r)+1}{\alpha q(r)}=\frac{1}{\alpha}=\tau .
$$

Let us prove the inequality $\underline{n}(\Lambda) \geqslant \tau$. In view of $(1)$, it is sufficient to show that $\underline{n}_{0}(\Lambda) \geqslant \tau$. We fix $\varepsilon>0$. In accordance with the hypothesis of the lemma and the definition of $\underline{n}_{0}(\widetilde{\Lambda})$ we find $\delta_{0}>0$ such that

$$
\lim _{r \rightarrow \infty} \frac{n(r, \widetilde{\Lambda})-n\left(\left(1-\delta^{\prime}\right) r, \widetilde{\Lambda}\right)}{\delta^{\prime} r} \geqslant \underline{n}_{0}(\widetilde{\Lambda})-\varepsilon \geqslant \tau-\varepsilon, \quad \delta^{\prime} \in\left(0, \delta_{0}\right) .
$$

Let $r>0$. By construction, $\Lambda_{p} \subset \widetilde{\Lambda}_{p}$. We denote by $p(r)$ the maximal index such that $\alpha p(r) \leqslant r$ and $\Lambda_{p(r)}$ is a proper subset of $\widetilde{\Lambda}_{p(r)}$. We can assume that for large $r$ such index exists since otherwise sequences $\Lambda$ and $\widetilde{\Lambda}$ coincide. Then the required inequality holds by the condition: $\underline{n}_{0}(\Lambda)=\underline{n}_{0}(\widetilde{\Lambda}) \geqslant \tau$.

We fix $\delta \in\left(0, \delta_{0}\right)$. We choose a sequence $r_{j} \rightarrow \infty$ such that

$$
\lim _{r \rightarrow \infty} \frac{n(r, \Lambda)-n((1-\delta) r, \Lambda)}{\delta r}=\lim _{j \rightarrow \infty} \frac{n\left(r_{j}, \Lambda\right)-n\left((1-\delta) r_{j}, \Lambda\right)}{\delta r_{j}} .
$$

In accordance with the construction and the choice of numbers $p(r)$ and $q(r)$, the intersections of the semi-interval $[\alpha p(r), \alpha q(r))$ with sets $\Lambda$ and $\widetilde{\Lambda}$ coincide. This is why the identity

$$
n(\alpha q(r), \Lambda)-n(\tilde{r}, \Lambda)=n(\alpha q(r), \widetilde{\Lambda})-n(\tilde{r}, \widetilde{\Lambda}), \quad \alpha p(r) \leqslant \tilde{r}<\alpha q(r)
$$

holds true. Let $\delta^{\prime} \in(0, \delta)$. Then by the definition of $q(r)$ there exists $r\left(\delta^{\prime}\right)>0$ such that $\left(1-\delta^{\prime}\right) r^{\prime} \geqslant(1-\delta) r$ as $r \geqslant r\left(\delta^{\prime}\right)$, where $r^{\prime}=\alpha q(r)$. If $\alpha p\left(r_{j(k)}\right) \leqslant(1-\delta) r_{j(k)}$ for some subsequence $\left\{r_{j(k)}\right\}$, by (3)-(5) we obtain

$$
\begin{aligned}
\lim _{r \rightarrow \infty} & \frac{n(r, \Lambda)-n((1-\delta) r, \Lambda)}{\delta r}=\lim _{k \rightarrow \infty} \frac{n\left(r_{j(k)}, \Lambda\right)-n\left((1-\delta) r_{j(k)}, \Lambda\right)}{\delta r_{j(k)}} \\
\geqslant & \lim _{k \rightarrow \infty} \frac{n\left(\alpha q\left(r_{j(k)}\right), \Lambda\right)-n\left((1-\delta) r_{j(k)}, \Lambda\right)}{\delta r_{j(k)}}=\lim _{r \rightarrow \infty} \frac{\left.\alpha q\left(r_{j(k)}\right), \widetilde{\Lambda}\right)-n\left((1-\delta) r_{j(k)}, \widetilde{\Lambda}\right)}{\delta r_{j(k)}} \\
\geqslant & \lim _{r \rightarrow \infty} \frac{n(\alpha q(r), \widetilde{\Lambda})-n((1-\delta) r, \widetilde{\Lambda})}{\delta r} \geqslant \frac{\delta^{\prime}}{\delta} \frac{\lim _{r \rightarrow \infty} \frac{r^{\prime}\left(n\left(r^{\prime}, \widetilde{\Lambda}\right)-n\left(\left(1-\delta^{\prime}\right) r^{\prime}, \widetilde{\Lambda}\right)\right.}{r \delta^{\prime} r^{\prime}}}{\delta^{\prime} r^{\prime}} \geqslant \frac{\delta^{\prime}}{\delta} \lim _{r^{\prime} \rightarrow \infty} \frac{n(\alpha q(r), \widetilde{\Lambda})-n\left(\left(1-\delta^{\prime}\right) r^{\prime}, \widetilde{\Lambda}\right)}{\delta} \geqslant \frac{\delta^{\prime}}{\delta}(\tau-\varepsilon) .
\end{aligned}
$$

Since the latter inequality holds for each $\delta^{\prime} \in(0, \delta)$, then

$$
\lim _{r \rightarrow \infty} \frac{n(r, \Lambda)-n((1-\delta) r, \Lambda)}{\delta r} \geqslant \tau-\varepsilon
$$

Thus, we can assume that $\alpha p\left(r_{j}\right)>(1-\delta) r_{j}$ for each $j \geqslant 1$. Passing to a subsequence, we can also suppose that

$$
\lim _{j \rightarrow \infty} \frac{n\left(r_{j}, \Lambda\right)-n\left(\alpha p\left(r_{j}\right), \Lambda\right)}{\delta r_{j}}=\lim _{j \rightarrow \infty} \frac{n\left(r_{j}, \Lambda\right)-n\left(\alpha p\left(r_{j}\right), \Lambda\right)}{\delta r_{j}} .
$$

Hence, we have

$$
\begin{aligned}
& \lim _{j \rightarrow \infty} \frac{n\left(r_{j}, \Lambda\right)-n\left(\alpha p\left(r_{j}\right), \Lambda\right)+n\left(\alpha p\left(r_{j}\right), \Lambda\right)-n\left((1-\delta) r_{j}, \Lambda\right)}{\delta r_{j}} \\
& \quad=\lim _{j \rightarrow \infty} \frac{n\left(r_{j}, \Lambda\right)-n\left(\alpha p\left(r_{j}\right), \Lambda\right)}{\delta r_{j}}+\lim _{j \rightarrow \infty} \frac{n\left(\alpha p\left(r_{j}\right), \Lambda\right)-n\left((1-\delta) r_{j}, \Lambda\right)}{\delta r_{j}} .
\end{aligned}
$$


Let us estimate separately the terms in (7). Since $\alpha p\left(r_{j}\right) \in\left((1-\delta) r_{j}, r_{j}\right)$, passing to a subsequence we can assume that $p\left(r_{j}\right) / r_{j}$ converges to some $\gamma$, at that, $\alpha \gamma \in[1-\delta, 1]$. We proceed to the second term in (7). By the choice of number $p(r)$, set $\Lambda_{p\left(r_{j}\right)}$ is a proper subset of $\widetilde{\Lambda}_{p\left(r_{j}\right)}$. Then by construction the identity $n\left(\alpha p\left(r_{j}\right), \Lambda\right)=p\left(r_{j}\right)$ holds true. Moreover, in accordance with the above restriction, we have the inequality $n\left(\alpha m\left(r_{j}\right), \Lambda\right) \leqslant m\left(r_{j}\right)$, where $m\left(r_{j}\right)$ is a minimal natural number such that $\alpha m\left(r_{j}\right) \geqslant(1-\delta) r_{j}$. Therefore,

$$
\lim _{j \rightarrow \infty} \frac{n\left(\alpha p\left(r_{j}\right), \Lambda\right)-n\left((1-\delta) r_{j}, \Lambda\right)}{\delta r_{j}} \geqslant \lim _{j \rightarrow \infty} \frac{p\left(r_{j}\right)-m\left(r_{j}\right)}{\delta r_{j}}=\frac{\gamma}{\delta}-\frac{1-\delta}{\alpha \delta} .
$$

If $\alpha \gamma=1$, by (8), (7) and (4) we obtain

$$
\lim _{r \rightarrow \infty} \frac{n(r, \Lambda)-n((1-\delta) r, \Lambda)}{\delta r} \geqslant \tau .
$$

Let $\alpha \gamma<1$ and $\tilde{\delta} \in(0,1-\alpha \gamma) \subset(0, \delta)$. Then there exists an index $j_{0}$ such that $\alpha p\left(r_{j}\right) \leqslant$ $(1-\tilde{\delta}) r_{j}^{\prime}, j \geqslant j_{0}$, where $r_{j}^{\prime}=\alpha q\left(r_{j}\right)$. This is why in view of (5) and (3) we obtain

$$
\begin{aligned}
\lim _{j \rightarrow \infty} \frac{n\left(r_{j}, \Lambda\right)-n\left(\alpha p\left(r_{j}, \delta\right), \Lambda\right)}{\delta r_{j}} & \geqslant \lim _{j \rightarrow \infty} \frac{n\left(\alpha q\left(r_{j}\right), \Lambda\right)-n\left((1-\tilde{\delta}) r_{j}^{\prime}, \Lambda\right)}{\delta r_{j}} \\
& =\frac{\tilde{\delta}}{\delta} \lim _{j \rightarrow \infty} \frac{r_{j}^{\prime}\left(n\left(r_{j}^{\prime}, \widetilde{\Lambda}\right)-n\left((1-\tilde{\delta}) r_{j}^{\prime}, \widetilde{\Lambda}\right)\right)}{r_{j} \tilde{\delta} r_{j}^{\prime}} \geqslant \frac{\tilde{\delta}}{\delta}(\tau-\varepsilon) .
\end{aligned}
$$

By (4), (7), (8) it yields

$$
\lim _{r \rightarrow \infty} \frac{n(r, \Lambda)-n((1-\delta) r, \Lambda)}{\delta r} \geqslant \frac{\gamma}{\delta}-\frac{1-\delta}{\alpha \delta}+\frac{\tilde{\delta}}{\delta}(\tau-\varepsilon) .
$$

Since $\alpha=1 / \tau$ and $\tilde{\delta}$ can be arbitrarily close to $1-\alpha \gamma<\delta$, then

$$
\lim _{r \rightarrow \infty} \frac{n(r, \Lambda)-n((1-\delta) r, \Lambda)}{\delta r} \geqslant \frac{\alpha \gamma}{\delta} \tau-\frac{1-\delta}{\delta} \tau+\frac{1-\alpha \gamma}{\delta}(\tau-\varepsilon)=\tau-\frac{1-\alpha \gamma}{\delta} \varepsilon \geqslant \tau-\varepsilon .
$$

Thus, in view of (9), (6) and the arbitrariness of number $\varepsilon>0$ we obtain the inequality $\underline{n}_{0}(\Lambda) \geqslant \tau$. By $(1)$ and $(2)$ it completes the proof.

Let $\Lambda=\left\{\lambda_{k}\right\}_{k=1}^{\infty}$ and $r>0$. We define

$$
V(r, \Lambda)=\sum_{0<\left|\lambda_{k}\right|<r} \frac{1}{\lambda_{k}} .
$$

In what follows we consider only real sequences $\Lambda$ and we represent them as $\Lambda=\Omega \cup \Xi$, where $\Omega=\left\{\omega_{k}\right\}_{k=1}^{\infty}$ and $\Xi=\left\{\xi_{k}\right\}_{k=1}^{\infty}$ are taken in the ascending order of their absolute values. These sequences consists of non-negative and negative terms of $\Lambda$.

Lemma 3. Let $\Lambda=\Omega \cup \Xi$, where $\Omega$ and $\Xi$ have the same density $\tau$. Then for each $\varepsilon>0$ there exists $r(\varepsilon)$ such that for each $r_{2}>r_{1}>r(\varepsilon)$ the inequality

$$
\left|V\left(r_{2}, \Lambda\right)-V\left(r_{1}, \Lambda\right)\right| \leqslant \ln \left(r_{2} / r_{1}\right)+\varepsilon
$$

holds true.

Proof. Without loss of generality we can assume that $\omega_{k} \neq 0, k \geqslant 1$. In accordance with Euler's representation we have

$$
\sum_{k=1}^{n} \frac{1}{k}=\ln n+\beta+\varepsilon(n)
$$


where $\beta$ is Euler constant and $\varepsilon(n) \rightarrow 0$ as $n \rightarrow \infty$. By the assumption, $\Omega$ and $\Xi$ have density $\tau$, i.e., the identities

$$
\begin{array}{ll}
\omega_{k}=\left|\omega_{k}\right|=k /\left(\tau+\delta^{\prime}(k)\right), & \xi_{k}=-\left|\xi_{k}\right|=-k /\left(\tau+\delta^{\prime \prime}(k)\right) \\
n(r, \Omega)=\tau r+\varepsilon^{\prime}(r) r, & n(r, \Xi)=\tau r+\varepsilon^{\prime \prime}(r) r,
\end{array}
$$

hold true, where $\delta^{\prime}(k), \delta^{\prime \prime}(k) \rightarrow 0$ as $k \rightarrow \infty$ and $\varepsilon^{\prime}(r), \varepsilon^{\prime \prime}(r) \rightarrow 0$ as $r \rightarrow \infty$. We fix $\tilde{\varepsilon}>0$. We choose index $m$ such that

$$
\left|\delta^{\prime}(k)\right| \leqslant \tilde{\varepsilon}, \quad\left|\delta^{\prime \prime}(k)\right| \leqslant \tilde{\varepsilon}, \quad|\varepsilon(n)| \leqslant \tilde{\varepsilon}, \quad k, n \geqslant m .
$$

We choose $r(\tilde{\varepsilon})>0$ so that

$$
n\left(r_{1}, \Omega\right) \geqslant m, n\left(r_{1}, \Xi\right) \geqslant m,\left|\ln \frac{\tau+\varepsilon^{\prime}\left(r_{2}\right)}{\tau+\varepsilon^{\prime}\left(r_{1}\right)}\right| \leqslant \tilde{\varepsilon},\left|\ln \frac{\tau+\varepsilon^{\prime \prime}\left(r_{2}\right)}{\tau+\varepsilon^{\prime \prime}\left(r_{1}\right)}\right| \leqslant \tilde{\varepsilon}, r_{2}>r_{1}>r(\tilde{\varepsilon}) .
$$

Then by (10)-(14) we obtain

$$
\begin{aligned}
\mid V\left(r_{2}, \Lambda\right) & -V\left(r_{1}, \Lambda\right)|=| \sum_{k=n\left(r_{1}, \Omega\right)+1}^{n\left(r_{2}, \Omega\right)} \frac{\tau+\delta^{\prime}(k)}{k}-\sum_{k=n\left(r_{1}, \Xi\right)+1}^{n\left(r_{2}, \Xi\right)} \frac{\tau+\delta^{\prime \prime}(k)}{k} \mid \\
& \leqslant\left|\sum_{k=n\left(r_{1}, \Omega\right)+1}^{n\left(r_{2}, \Omega\right)} \frac{\tau}{k}-\sum_{k=n\left(r_{1}, \Xi\right)+1}^{n\left(r_{2}, \Xi\right)} \frac{\tau}{k}\right|+\sum_{k=n\left(r_{1}, \Omega\right)+1}^{n\left(r_{2}, \Omega\right)} \frac{\left|\delta^{\prime}(k)\right|}{k}+\sum_{k=n\left(r_{1}, \Xi\right)+1}^{n\left(r_{2}, \Xi\right)} \frac{\left|\delta^{\prime \prime}(k)\right|}{k} \\
& \leqslant \tau\left|\ln \frac{n\left(r_{2}, \Omega\right)}{n\left(r_{1}, \Omega\right)}-\ln \frac{n\left(r_{2}, \Xi\right)}{n\left(r_{1}, \Xi\right)}\right|+4 \tau \tilde{\varepsilon}+\tilde{\varepsilon} \ln \frac{n\left(r_{2}, \Omega\right)}{n\left(r_{1}, \Omega\right)}+\tilde{\varepsilon} \ln \frac{n\left(r_{2}, \Xi\right)}{n\left(r_{1}, \Xi\right)}+4 \tilde{\varepsilon}^{2} \\
& \leqslant 6 \tau \tilde{\varepsilon}+2 \tilde{\varepsilon} \ln \frac{r_{2}}{r_{1}}+6 \tilde{\varepsilon}^{2}, \quad r_{2}>r_{1}>r(\tilde{\varepsilon}) .
\end{aligned}
$$

It follows easily the required inequality. The proof is complete.

Lemma 4. Let $\Lambda=\Omega \cup \Xi$, where $\Omega$ and $\Xi$ have the same density $\tau$. Then there exists a set of zero density $T \subset \Lambda$ such that

$$
\lim _{r \rightarrow \infty}(V(r, \Lambda)-V(r, T))=0 .
$$

Proof. If $\tau=0$, then $\Lambda$ has the zero density. In this case the statement of the lemma is trivial since as $T$ we can take $\Lambda$.

Let $\tau>0$. We seek sequence $T \subset \Lambda, T=\left\{t_{p}\right\}$ as the union $T=\cup_{m \geqslant 1} T_{m}$, where $T_{m}=$ $\left\{t_{p}\right\}_{p=p(m)}^{p(m+1)-1}$. We construct sets $T_{m}$ by induction. Let $m=1$. As $T_{1}=\left\{t_{p}\right\}_{p=p(1)=1}^{p(2)-1}$ we take a set formed by all the elements in $\Lambda$ belonging to the interval $(-2,2)$. We suppose that we have constructed sets $T_{m}$ for each $m<l$. Let us define $T_{l}$. We consider two cases.

1) $V\left(2^{l}, \Lambda\right)-\sum_{p=1}^{p(l)-1} 1 / t_{p} \geqslant 0$.

a) If

$$
V\left(2^{l}, \Lambda\right)-\sum_{p=1}^{p(l)-1} \frac{1}{t_{p}}-\sum_{2^{l-1} \leqslant \omega_{k}<2^{l}} \frac{1}{\omega_{k}} \geqslant 0,
$$

then as $T_{l}$ we take the set (probably empty) of all the elements in $\Omega$ belonging to the semiinterval $\left[2^{l-1}, 2^{l}\right)$.

b) Let

$$
V\left(2^{l}, \Lambda\right)-\sum_{p=1}^{p(l)-1} \frac{1}{t_{p}}-\sum_{2^{l-1} \leqslant \omega_{k}<2^{l}} \frac{1}{\omega_{k}}<0 .
$$


Then semi-interval $\left[2^{l-1}, 2^{l}\right)$ contains points $\omega_{k}$ of sequence $\Omega$. By $k(l)$ we denote the minimal of indices $k$ for which $\omega_{k} \geqslant 2^{l-1}$. As $T_{l}$ we take the set of the elements $\omega_{k(l)}, \ldots, \omega_{k^{\prime}(l)}$, where $k^{\prime}(l)$ is the minimal index satisfying the inequality

$$
V\left(2^{l}, \Lambda\right)-\sum_{p=1}^{p(l)-1} \frac{1}{t_{p}}-\sum_{k=k(l)}^{k^{\prime}(l)} \frac{1}{\omega_{k}}<0 .
$$

By the choice of $k^{\prime}(l)$, the point $\omega_{k^{\prime}(l)}$ together with all the elements in set $T_{l}$ belong the semi-interval $\left[2^{l-1}, 2^{l}\right)$.

2) $V\left(2^{l}, \Lambda\right)-\sum_{p=1}^{p(l)-1} 1 / t_{p}<0$.

a) If

$$
V\left(2^{l}, \Lambda\right)-\sum_{p=1}^{p(l)-1} \frac{1}{t_{p}}-\sum_{-2^{l}<\xi_{n} \leqslant-2^{l-1}} \frac{1}{\xi_{n}}<0,
$$

as $T_{l}$ we take the set of all the elements $\Xi$ lying in the semi-interval $\left(-2^{l},-2^{l-1}\right]$.

b) Let

$$
V\left(2^{l}, \Lambda\right)-\sum_{p=1}^{p(l)-1} \frac{1}{t_{p}}-\sum_{-2^{l}<\xi_{n} \leqslant-2^{l-1}} \frac{1}{\xi_{n}} \geqslant 0 .
$$

Then semi-interval $\left(-2^{l},-2^{l-1}\right]$ contains points $\xi_{n}$ of sequence $\Xi$. By $n(l)$ we denote the minimal of indices $n$ satisfying $\xi_{n} \leqslant-2^{l-1}$. As $T_{l}$, we take the set of the elements $\xi_{n(l)}, \ldots, \xi_{n^{\prime}(l)}$, where $n^{\prime}(l)$ is the minimal index satisfying the estimate

$$
V\left(2^{l}, \Lambda\right)-\sum_{p=1}^{p(l)-1} \frac{1}{t_{p}}-\sum_{n=n(l)}^{n^{\prime}(l)} \frac{1}{\xi_{n}} \geqslant 0 .
$$

By the choice of $n^{\prime}(l)$, point $\omega_{k^{\prime}(l)}$ together with all the elements in $T_{l}$ belong to the semi-interval $\left[2^{l-1}, 2^{l}\right)$.

Thus, sequence $T$ is defined completely. We note that by construction, one of the following possibilities occurs:

a) the quantity $V\left(2^{m}, \Lambda\right)-V\left(2^{m}, T\right)$ preserves the sign (number 0 is assigned with sign "+") while passing from $m=l-1$ to $m=l(l>1)$ and thus, the inequality

$$
\left|V\left(2^{l}, \Lambda\right)-V\left(2^{l}, T\right)\right| \leqslant\left|V\left(2^{l-1}, \Lambda\right)-V\left(2^{l-1}, T\right)\right|
$$

holds true, while set $T_{l}$ consists of all the elements in $\Lambda$ lying in the annulus $B\left(0,2^{l}\right) \backslash B\left(0,2^{l-1}\right)$ and having the same sign as $V\left(2^{l}, \Lambda\right)-V\left(2^{l}, T\right)$.

b) quantity $V\left(2^{m}, \Lambda\right)-V\left(2^{m}, T\right)$ changes the sign while passing from $m=l-1$ to $m=l$ and thus, the estimate

$$
\left|V\left(2^{l}, \Lambda\right)-V\left(2^{l}, T\right)\right| \leqslant 1 / \omega_{k^{\prime}(l)}\left(1 /\left|\xi_{n^{\prime}(l)}\right|\right) \leqslant 1 / 2^{l-1}
$$

holds true. It is implied by the choice of indices $k^{\prime}(l)$ and $n^{\prime}(l)$.

Suppose that for some index $m(0)$ the quantity $V\left(2^{m}, \Lambda\right)-V\left(2^{m}, T\right)$ preserves the sign for all $m \geqslant m(0)$, for instance, "+". Then the part of sequence $T$ formed by the elements lying outside the circle $B\left(0,2^{m(0)}\right)$ coincides with the corresponding part of sequence $\Omega$. Then by (15), the series $\sum 1 / \xi_{n}$ converges. It means that $n / \xi_{n} \rightarrow 0$ as $n \rightarrow \infty$, i.e., sequence $\Xi$ has the zero density. It contradicts to the inequality $\tau>0$.

Thus, there exists a sequence of indices $m(j), j \geqslant 1$, such that the quantity $V\left(2^{m}, \Lambda\right)-$ $V\left(2^{m}, T\right)$ changes the sign while passing from $m=m(j)$ to $m=m(j)+1$. Then it follows from (15) and (16) that

$$
\left|V\left(2^{m}, \Lambda\right)-V\left(2^{m}, T\right)\right| \rightarrow 0, \quad m \rightarrow \infty
$$


Let us prove that $T$ has the zero density. Let $s_{m}$ be then number of the elements in $T_{m}$. By construction, all of them have the same sign and lie in the annulus $B\left(0,2^{m}\right) \backslash B\left(0,2^{m-1}\right)$. Therefore, by (17) and Lemma 3 we obtain

$$
\begin{aligned}
\frac{s_{m}}{2^{m}} \leqslant & \left|\sum_{p=p(m)}^{p(m+1)-1} \frac{1}{t_{p}}\right|=\left|V\left(2^{m}, T\right)-V\left(2^{m-1}, T\right)\right| \leqslant\left|V\left(2^{m}, \Lambda\right)-V\left(2^{m-1}, \Lambda\right)\right| \\
& +\left|V\left(2^{m}, \Lambda\right)-V\left(2^{m}, T\right)\right|+\left|V\left(2^{m-1}, \Lambda\right)-V\left(2^{m-1}, T\right)\right| \rightarrow 0, \quad m \rightarrow \infty .
\end{aligned}
$$

We fix $\varepsilon>0$. Then there exists an index $m(\varepsilon)$ such that

$$
s_{m} / 2^{m} \leqslant \varepsilon, \quad m \geqslant m(\varepsilon) .
$$

Let $r>2^{m(\varepsilon)}$ and $n$ be the minimal of the indices for which $r \leqslant 2^{n}$. Then by (18)

$$
\begin{aligned}
\frac{n(r, T)}{r} \leqslant & \frac{n\left(2^{m(\varepsilon)}, T\right)}{r}+\frac{n\left(2^{n}, T\right)-n\left(2^{m(\varepsilon)}, T\right)}{2^{n-1}}=\frac{n\left(2^{m(\varepsilon)}, T\right)}{r} \\
& +\frac{s_{m(\varepsilon)+1}+s_{m(\varepsilon)+2}+\cdots+s_{n}}{2^{n-1}} \leqslant \frac{n\left(2^{m(\varepsilon)}, T\right)}{r}+2 \varepsilon\left(1+\frac{1}{2}+\cdots+\frac{1}{2^{n-m(\varepsilon)-1}}\right) .
\end{aligned}
$$

It follows that $\bar{n}(T) \leqslant 2 \varepsilon$. Since $\varepsilon>0$ is arbitrary and $\underline{n}(T) \geqslant 0$, it leads to the required identity $n(T)=0$.

It remains to show that $V(r, \Lambda)-V(r, T) \rightarrow 0$ as $r \rightarrow \infty$. Let $r>2$. We choose index $m$ such that $2^{m} \leqslant r<2^{m+1}$. As it has been proved above, $T$ has the zero density. This is why for sequence $T$, as well as for $\Lambda$, Lemma 3 holds true. Then by (17) we have

$$
\begin{gathered}
|V(r, \Lambda)-V(r, T)| \leqslant\left|V\left(2^{m}, \Lambda\right)-V\left(2^{m}, T\right)\right|+\left|V(r, \Lambda)-V\left(2^{m}, \Lambda\right)\right|+\left|V(r, T)-V\left(2^{m}, T\right)\right| \\
\leqslant\left|V\left(2^{m}, \Lambda\right)-V\left(2^{m}, T\right)\right|+2 \varepsilon(r)(\ln 2+1) \rightarrow 0, \quad r \rightarrow \infty .
\end{gathered}
$$

The proof is complete.

We recall that set $\Lambda=\Omega \cup \Xi$ is called properly distributed (see [2, Ch. II, Sect. 1]), if sequences $\Omega$ and $\Xi$ have densities and there exists $\lim _{r \rightarrow \infty} V(r, \Lambda)$.

Theorem 1. Let $\tau \geqslant 0$ and $\widetilde{\Lambda}=\widetilde{\Omega} \cup \widetilde{\Xi}$ be such that $\underline{n}(\widetilde{\Omega}) \geqslant \tau$ and $\underline{n}(\widetilde{\Xi}) \geqslant \tau$. Then there exists a properly distributed set $\Lambda \subset \widetilde{\Lambda}, \Lambda=\Omega \cup \Xi$, where $\Omega$ and $\Xi$ have the same density $\tau$ and $\lim _{r \rightarrow \infty} V(r, \Lambda)=0$.

Proof. In accordance with Lemma 2, there exists a sequence $\Lambda^{\prime} \subset \widetilde{\Lambda}, \Lambda^{\prime}=\Omega^{\prime} \cup \Xi^{\prime}$ such that $n\left(\Omega^{\prime}\right)=n\left(\Xi^{\prime}\right)=\tau$. Then by Lemma 4 there exists a sequence $T \subset \Lambda^{\prime}$ satisfying condition $\lim _{r \rightarrow \infty}\left(V\left(r, \Lambda^{\prime}\right)-V(r, T)\right)=0$. Thus, $\Lambda=\Lambda^{\prime} \backslash T$ possesses all the required properties. The proof is complete.

Let us consider some applications of Theorem 1.

Let $f$ be an entire function of exponential type (i.e., there exist $A, B>0$ such that the estimate $|f(z)| \leqslant A+B|z|, z \in \mathbb{C}$ holds true). The upper indicator of $f$ is the function

$$
h_{f}(\lambda)=\varlimsup_{t \rightarrow \infty} \ln |f(t \lambda)| / t, \quad \lambda \in \mathbb{C} .
$$

Indicator $h_{f}$ is a convex order one positively homogeneous function which coincides with the complex support function $H_{K}$ of some convex compact set $K$ (in other words, with the usual support function of the compact set complex conjugate with $K$ ) called adjoint diagram of $f$ (see, for instance, [3, Ch. I, Sect. 5, Thm. 5.4]):

$$
h_{f}(\lambda)=H_{K}(\lambda)=\sup _{z \in K} \operatorname{Re}(\lambda z), \quad \lambda \in \mathbb{C} .
$$


Function $f$ is said to have a regular growth (see $[2$, Ch. III]) if

$$
h_{f}(\lambda)=\lim _{t \rightarrow \infty, t \notin E} \ln |f(t \lambda)| / t, \quad \lambda \in \mathbb{C},
$$

where $E$ is a set of relative zero measure on the ray $(0,+\infty)$, i.e., the Lebesgue measure of its intersection with the interval $(0, r)$ is infinitesimally small w.r.t. $r$ as $r \rightarrow+\infty$.

Let $K$ be a convex compact set. The points $z(\varphi)$ being the intersection of support line $l(\varphi)=\left\{z: \operatorname{Re}\left(z e^{i \varphi}=H_{K}\left(e^{i \varphi}\right)\right\}\right.$ and the boundary $\partial K$ are called support points of line $l(\varphi)$.

In accordance of $[2, \mathrm{Ch}$. III, Thm. 4], function $f$ has a regular growth if and only if its multiple zero set $\Lambda$ (i.e., each zero of $f$ appears in $\Lambda$ counting its multiplicity) is properly distributed. At that, if $\Lambda$ is real $(\Lambda=\Omega \cup \Xi)$, the identities

$$
\begin{aligned}
& n(\Omega)=S_{K}\left(\varphi_{1}, \varphi_{2}\right) / 2 \pi, \quad-\pi / 2<\varphi_{1}<\varphi_{2}<\pi / 2, \\
& n(\Xi)=S_{K}\left(\varphi_{1}, \varphi_{2}\right) / 2 \pi, \quad \pi / 2<\varphi_{1}<\varphi_{2}<3 \pi / 2,
\end{aligned}
$$

hold true, see [2, Ch. II, Sect. 1, Formula (2.07)]. Here $K$ is the adjoint diagrma, and $S_{K}\left(\varphi_{1}, \varphi_{2}\right)$ is the length of boundary $\partial K$ (measured in the clockwise direction) between support points $z\left(\varphi_{1}\right)$ and $z\left(\varphi_{2}\right)$. We observe that for all mentioned $\varphi_{1}, \varphi_{2}$ quantity $S_{K}\left(\varphi_{1}, \varphi_{2}\right)$ is constant. It is possible if and only if $K$ is the vertical segment of length $2 \pi \tau$, where $\tau=n(\Omega)=n(\Xi)$.

Theorem 2. Let $f$ be an entire function of exponential type with a real zero set $\widetilde{\Lambda}=\widetilde{\Omega} \cup \widetilde{\Xi}$. Then the following statements are equivalent:

1) The inequalities $\underline{n}(\widetilde{\Omega}) \geqslant \tau, \underline{n}(\widetilde{\Xi}) \geqslant \tau$ hold true.

2) The representation $f=f_{1} f_{2}$ is valid, where $f_{1}, f_{2}$ are entire function of exponential type, $f_{1}$ is a function of regular growth, its adjoint diagram is a vertical segment of length $2 \pi \tau$ and $h_{f_{2}} \equiv h_{f}-h_{f_{1}}$.

Proof. Assume that Statement 1) holds true. Then by Theorem 1 there exists a properly distributed set $\Lambda \subset \widetilde{\Lambda}, \Lambda=\Omega \cup \Xi$, where $\Omega$ and $\Xi$ have the same density $\tau$. By $f_{1}$ we denote the canonical function of set $\Lambda$. It has exponential type and regular growth (see $[2$, Ch. II, Thm. 4]). At that, as it was mentioned above, the adjoint diagram $f_{1}$ coincides with the vertical segment of length $2 \pi \tau$. We let $f_{2}=f / f_{1}$. Since the zero set of $f_{1}$ is a part of zero set of $f$, function $f_{2}$ is entire. Then by Corollary 2 of Theorem 5 in $\left[2\right.$, Ch. III] the identity $h_{f_{2}} \equiv h_{f}-h_{f_{1}}$ holds true. In particular, it implies that $f_{2}$ is of exponential type.

Assume that Statement 2 holds true. Then zero set $\Lambda=\Omega \cup \Xi$ of function $f_{1}$ satisfies the identities $n(\Omega)=n(\Xi)=\tau$. Since $\Lambda$ is a part of $\widetilde{\Lambda}$, it yields $\underline{n}(\widetilde{\Omega}) \geqslant \tau, \underline{n}(\widetilde{\Xi}) \geqslant \tau$. The proof is complete.

Theorem 3. Let $f$ be an entire function of exponential type with real zero set $\widetilde{\Lambda}=\widetilde{\Omega} \cup \widetilde{\Xi}$, where $\widetilde{\Omega}$ and $\widetilde{\Xi}$ possess densities. Then the representation $f=f_{1} f_{2}$ holds true, where $f_{1}, f_{2}$ are entire function of exponential type, $f_{1}$ is a function of regular growth, its adjoint diagram is a vertical segment, $h_{f_{2}} \equiv h_{f}-h_{f_{1}}$ and the zero set of $f_{2}$ has the zero density.

Proof. Let us show first that the densities of $\widetilde{\Omega}$ and $\widetilde{\Xi}$ are same. Since $f$ has an exponential type, by the Lindelöf's theorem (see [2, Ch. I, Thm. 15]) there exists $c>0$ such that $|V(r, \widetilde{\Lambda})| \leqslant c$, $r>0$. Let $n(\widetilde{\Omega})=\tau$ and $\underline{n}(\widetilde{\Xi})=\gamma$. Assume that $\tau \neq \gamma$, for instance, $\tau>\gamma$ (the case $\tau<\gamma$ is studied in the same way). As in Lemma 3, we have (without loss of generality we can assume 
that $0 \notin \widetilde{\Omega})$ :

$$
\begin{aligned}
V(r, \widetilde{\Lambda})= & \sum_{k=1}^{n(r, \widetilde{\Omega})} \frac{\tau+\delta^{\prime}(k)}{k}-\sum_{k=1}^{n(r, \widetilde{\Xi})} \frac{\gamma+\delta^{\prime \prime}(k)}{k}=\tau \ln n(r, \widetilde{\Omega})-\gamma \ln n(r, \widetilde{\Xi}) \\
& +\sum_{k=1}^{n(r, \widetilde{\Omega})} \frac{\delta^{\prime}(k)}{k}-\sum_{k=1}^{n(r, \widetilde{\Xi})} \frac{\delta^{\prime \prime}(k)}{k}+\varepsilon(n(r, \widetilde{\Omega}))-\varepsilon(n(r, \widetilde{\Xi})), \\
n(r, \widetilde{\Omega})= & \tau r+\varepsilon^{\prime}(r) r, \quad n(r, \widetilde{\Xi})=\tau r+\varepsilon^{\prime \prime}(r) r,
\end{aligned}
$$

where $\varepsilon(k), \delta^{\prime}(k), \delta^{\prime \prime}(k) \rightarrow 0, k \rightarrow \infty$ and $\varepsilon^{\prime}(r), \varepsilon^{\prime \prime}(r) \rightarrow 0, r \rightarrow \infty$. We fix $\varepsilon>0$. We choose index $m$ such that $\left|\delta^{\prime}(k)\right| \leqslant \varepsilon,\left|\delta^{\prime \prime}(k)\right| \leqslant \varepsilon,|\varepsilon(n)| \leqslant \varepsilon, k, n \geqslant m$. Then we choose $r(\varepsilon)>0$ so that $n(r, \widetilde{\Omega}) \geqslant m, n(r, \widetilde{\Xi}) \geqslant m, r>r(\varepsilon)$. By (10) it implies

$$
\begin{aligned}
|V(r, \widetilde{\Lambda})| \geqslant & (\tau-\gamma) \ln r-\tau\left|\ln \left(\tau+\varepsilon \varepsilon^{\prime}(r)\right)\right|-\gamma\left|\ln \left(\gamma+\varepsilon^{\prime \prime}(r)\right)\right|-\sum_{k=1}^{m} \frac{\left|\delta^{\prime}(k)\right|+\left|\delta^{\prime \prime}(k)\right|}{k} \\
& -2 \varepsilon \ln r-\varepsilon\left|\ln \left(\tau+\varepsilon^{\prime}(r)\right)\right|-\varepsilon \varepsilon\left|\ln \left(\gamma+\varepsilon^{\prime \prime}(r)\right)\right|-2 \varepsilon-2 \beta, \quad r>r(\varepsilon) .
\end{aligned}
$$

It yields that $|V(r, \widetilde{\Lambda})| \rightarrow \infty$ as $r \rightarrow \infty$. It contradicts the boundedness of $|V(r, \widetilde{\Lambda})|$. Thus, sequences $\widetilde{\Omega}$ and $\widetilde{\Xi}$ have the same density $\tau$.

As in Theorem 2, the representation $f=f_{1} f_{2}$ holds true, where $f_{1}, f_{2}$ are entire functions of exponential type, $f_{1}$ has a regular growth, its adjoint diagram is a vertical segment of length $2 \pi \tau$, and $h_{f_{2}} \equiv h_{f}-h_{f_{1}}$. At that, $\widetilde{\Lambda}=\Lambda \cup \Lambda^{\prime}$, where $\Lambda=\Omega \cup \Xi, \Lambda^{\prime}$ are zero sets of functions $f_{1}, f_{2}$, respectively, and $n(\Omega)=n(\Xi)=\tau$. Since the densities of sequences $\widetilde{\Omega}$ and $\widetilde{\Xi}$ are also equal to $\tau$, we get

$$
\lim _{r \rightarrow \infty} \frac{n\left(r, \Lambda^{\prime}\right)}{r}=\lim _{r \rightarrow \infty} \frac{n(r, \widetilde{\Lambda})-n(r, \Lambda)}{r}=\lim _{r \rightarrow \infty} \frac{n(r, \widetilde{\Omega})+n(r, \widetilde{\Xi})-n(r, \Omega)-n(r, \Xi)}{r}=0 .
$$

The proof is complete.

Let $D$ be a convex set in $\mathbb{C}, H(D)$ is the space of functions analytic in $D$ with the topology of uniform convergence on compact subsets in $D$, and $H^{*}(D)$ is a strongly dual space for $H(D)$. By $P_{D}$ we denote the space of entire functions of exponential type whose adjoint diagrams lie in domain $D$. The Laplace transform $f(\lambda)=\nu(\exp (\lambda z))$ makes an isomorphism (see, for instance, [4, Ch. III, Sect. 12, Thm. 12.3]) between $H^{*}(D)$ and $P_{D}$.

Let $\Lambda=\left\{\lambda_{k}\right\}_{k=1}^{\infty}$ and $\mathcal{E}(\Lambda)=\left\{z^{n-1} \exp \left(\lambda_{k} z\right)\right\}_{n=1, k}^{n(k)}$, where we go over various points $\lambda_{k}$, and $n(k)$ is the multiplicity of $\lambda_{k}$ (i.e., the number of elements in sequence $\Lambda$ coinciding with $\left.\lambda_{k}\right)$. By the Hahn-Banach theorem, system $\mathcal{E}(\Lambda)$ is incomplete in space $H(D)$ if and only if there exists a non-zero functional $\nu \in H^{*}(D)$ vanishing on the elements of the system. Hence, the incompleteness of $\mathcal{E}(\Lambda)$ is equivalent to the existence of function $f \in P_{D}$ vanishing on the points $\lambda_{k}$ with multiplicity at least $n(k), k=1,2, \ldots$ If domain $D$ is non-empty, for the sake of convenience we assume that each system $\mathcal{E}(\Lambda)$ is complete in $H(D)$.

Let $\tau>0, I(\tau)=[-i \pi \tau, i \pi \tau]$, and $D(\tau)$ denotes a convex domain covered while segment $I(\tau)$ moves inside $D$. By the definition, the inclusion $D(\tau) \subset D$ holds true. Domain $D(\tau)$ is empty if none of the shifts of segment $I(\tau)$ lies in $D$. We obviously have the representation $D(\tau)=D^{\prime}(\tau)+I(\tau)$, where

$$
D^{\prime}(\tau)=\left\{z \in \mathbb{C}: \operatorname{Re}(z \lambda)<H_{D}(\lambda)-\pi \tau|\operatorname{Im} \lambda|, \quad \forall \lambda:|\lambda|=1\right\}
$$

$\pi \tau|\operatorname{Im} \lambda|$ is the support function of segment $I(\tau)$.

Theorem 4. Let $\tau>0, D$ be a convex domain and $\widetilde{\Lambda}=\widetilde{\Omega} \cup \widetilde{\Xi}$ is a real sequence such that $\underline{n}(\widetilde{\Omega}) \geqslant \tau, \underline{n}(\widetilde{\Xi}) \geqslant \tau$. System $\mathcal{E}(\widetilde{\Lambda})$ is complete in $H(D)$ if and only if it is complete in $H(D(\tau))$. 
Proof. Suppose that $\mathcal{E}(\widetilde{\Lambda})$ is incomplete in space $H(D(\tau))$. Then there exists an entire function $f$ of exponential type vanishing at the points $\lambda_{k}$ of the multiplicity at least $n(k), k=1,2, \ldots$, whose adjoint diagram lies in $D(\tau)$. Since $D(\tau) \subset D$, we have $f \in P_{D}$. It follows that $\mathcal{E}(\widetilde{\Lambda})$ is incomplete in space $H(D)$.

Suppose that $\mathcal{E}(\widetilde{\Lambda})$ is incomplete in $H(D)$. Then there exists $f \in P_{D}$ vanishing at points $\lambda_{k}$ of multiplicity at least $n(k), k=1,2, \ldots$ Let us show that $f \in P_{D(\tau)}$.

In accordance with Theorem 1 , there exists a properly distributed set $\Lambda \subset \widetilde{\Lambda}, \Lambda=\Omega \cup \Xi$, where $\Omega$ and $\Xi$ have the same density $\tau$. By $f_{1}$ we denote the canonical function of set $\Lambda$. It is of exponential type and regular growth, while its adjoint diagram $K_{1}$ is represented as $I(\tau)+z_{0}$ ( $z_{0}$ is some point in the plane). We let $f_{2}=f / f_{1}$. Since the zero set of $f_{1}$ is a part of zero set for $f$, function $f_{2}$ is entire. Then in accordance with Corollary 2 of Theorem 5 in [2, Ch. III] the identity $h_{f_{2}} \equiv h_{f}-h_{f_{1}}$ holds true. In particular, it follows that $f_{2}$ is of exponential type. Let $K$ and $K_{2}$ be adjoint diagrams of functions $f$ and $f_{2}$, respectively. Then

$$
H_{K_{2}} \equiv h_{f_{2}} \equiv h_{f}-h_{f_{1}} \equiv H_{K}-H_{K_{1}} .
$$

Thus, $H_{K} \equiv H_{K_{1}}+H_{K_{2}}$, i.e., $K=K_{1}+K_{2}=I(\tau)+z_{0}+K_{2} \subset D$. Hence, if $z \in K$, then $z$ belongs to a shift of segment $I(\tau)$ lying in domain $D$. It means that the inclusion $K \subset D(\tau)$ is true. It follows that $\mathcal{E}(\widetilde{\Lambda})$ is incomplete in $H(D(\tau))$. The proof is complete.

\section{BIBLIOGRAPHY}

1. A.S. Krivosheyev, O.A. Krivosheyeva. A closedness of set of Dirichlet series sum // Ufimskij Matem. Zhurn. 5:3, 96-120 (2013). [Ufa Math. J. 5:3, 94-117 (2013).]

2. B.Ya. Levin. Distribution of the zeros of entire functions. Fizmatgiz, Moscow (1956). [Mathematische Lehrbücher und Monographien. II. Abt. Band 14. Akademie-Verlag, Berlin (1962). (in German).]

3. A.F. Leont'ev. Entire functions. Exponential series. Nauka, Moscow (1983). (in Russian).

4. V.V. Napalkov. Convolution equations in multi-dimensional spaces. Nauka, Moscow (1982). (in Russian).

Aidar Irekovich Abdulnagimov,

Ufa State Aviation Technical University,

Karl Marx str., 12, bld. 1,

450000, Ufa, Russia

E-mail: buffonishe@mail.ru

Alexander Sergeevich Krivosheev, Institute of Mathematics CC USC RAS,

Chernyshevsky str., 112,

450008, Ufa, Russia

E-mail: kriolesya2006@yandex.ru 